\title{
The Number of Power Line Spans Accounting in Determining the Induced Voltage
}

\author{
Igor V. Belitsyn* \\ Polzunov Altai State Technical University \\ 46 Lenin, Barnaul, 656038, Russia
}

Received 29.11.2017, received in revised form 10.12.2018, accepted 27.02.2019

The article considers inductive interference components. A description of the induced voltage electromagnetic component is described in detail. The technique of the calculated wire suspension height determining based on the actual magnetic field distribution is presented. The technique for the number of power line spans determining is provided. Dependences of the magnetic field induction vector on the distance along one power line's span are obtained. Relationships of the magnetic field induction determining error depending on the number of adjacent spans are obtained. Recommendations for the number of spans determining, which must be taken into account when evaluating the magnetic component of the power line electromagnetic field are given.

Keywords: power transmission line, induced voltage, magnetic field, sag of a wire.

Citation: Belitsyn I.V. The number of power line spans accounting in determining the induced voltage, J. Sib. Fed. Univ. Eng. technol., 2019, 12(3), 346-355. DOI: 10.17516/1999-494X-0142.

\section{Учет числа пролетов воздушной линии электропередачи при определении наведенного напряжения}

И.В. Белицын

Алтайский государственный технический университет им. И.И. Ползунова Россия, 656038, Барнаул, пр. Ленина, 46

В статье рассмотрены составляющие индуктивных помех. Подробно представлено описание электромагнитной составляющей наведенного напряжения. Приведена методика определения расчетной высоты подвеса провода, основанная на фактическом распределении магнитного поля. Приведена методика определения числа пролетов воздушной линии электропередачи. Получены зависимости вектора индукиии магнитного поля от расстояния вдоль одного

(C) Siberian Federal University. All rights reserved

This work is licensed under a Creative Commons Attribution-NonCommercial 4.0 International License (CC BY-NC 4.0).

* Corresponding author E-mail address: b_i_w@mail.ru 
пролета. Получены зависимости относительной погрешности определения индукиии магнитного поля от количества смежных пролетов. Даны рекомендации по определению числа пролетов, которое необходимо учитывать при расчете магнитной составляющей электромагнитного поля, создаваемого линией электропередачи.

Ключевые слова: линия электропередачи, наведенное напряжение, магнитное поле, стрела провеса провода.

\section{Введение}

Воздушная линия электропередачи (ЛЭП) является источником кондуктивных и индуктивных помех. Расчет индуктивных помех необходим для обеспечения надежного функционирования устройств релейной защиты и автоматики, передачи информации по каналам связи, обеспечения безопасности при проведении работ на оборудовании и электрических сетях, находящихся в зоне влияния воздушной линии электропередачи.

На отключенную воздушную ЛЭП от функционирующих в непосредственной близости от нее соседних линий наводится напряжение, которое складывается с напряжением самой линии. Такое напряжение называется наведенным.

В связи с этим правила по охране труда при эксплуатации электроустановок (ПОТЭУ) предписывают комплекс защитных мероприятий, которые необходимы для обеспечения безопасности при проведении работ на воздушных ЛЭП. Отдельно отмечаются меры безопасности в случаях, когда заземление проводников не позволяет снизить значение наведенного потенциала на отключенной линии ниже 25 В.

Практика эксплуатации воздушных ЛЭП показывает, что каждый год обслуживающий персонал получает электротравмы вследствие наведенного напряжения, приводящего к протеканию через тело человека электрического тока. Появление наведенного напряжения связано с электростатическим и индукционным воздействием от воздушной линии, которая проходит в непосредственной близости, на отключенную ЛЭП.

Электромагнитное поле влияющей линии связано с ее напряжением, током, топологическими особенностями. Кроме этого, большое значение играет длина участка сближения влияющей и отключенной линий. На каждом проводнике отключенной линии наводится потенциал, который определяется суммой двух составляющих: электростатического и электромагнитного взаимодействий [1].

Электростатическая составляющая наведенного напряжения связана с взаимодействием электрической составляющей электромагнитного поля влияющей линии на рассматриваемый проводник отключенной. Значение этой составляющей, даже при соблюдении требований правил устройства электроустановок (ПУЭ), при параллельном прохождении линий зависит, в первую очередь, от напряжения на влияющей линии и коэффициента емкостной связи. Точное определение коэффициента емкостной связи возможно только в случае определения электрического поля влияющей линии с учетом всех ее топологических особенностей, в частности стелы провеса проводников. Электростатическая составляющая наведенного напряжения может быть снижена до безопасного значения на всей линии путем ее заземления в любом месте. При заземлении отключенной линии по обоим концам действие электростатической составляющей будет полностью нивелировано.

$$
-347-
$$


Электромагнитная составляющая наведенного напряжения связана с взаимодействием магнитной составляюей электромагнитного поля влияющей линии на рассматриваемый проводник отключенной. В этом случае решающее значение имеет коэффициент индуктивной связи и ток, протекающий по проводникам влияющей линии.

\section{Анализ модели расчета наведенного напряжения}

На основе методов, изложенных в [2], возможно уточнить типовую методику расчета наведенного напряжения.

Исходными данными для расчетов являются расстояния между осями трасс ВЛ на участках сближения, длина ВЛ, значения сопротивлений контуров заземления опор и подстанций, находящихся по концам линий и на границах участков, а также значения токов, которые могут протекать во влияющих ЛЭП. Для сетей произвольной топологии расчет значений наведенного напряжения на отключенной ЛЭП производится на основе упрощенной схемы замещения, полученной на основе П-образной схемы замещения. В этом случае воздушная линия электропередачи разделена на два участка с различными удельными наведенными ЭДС.

С практической точки зрения целесообразно учитывать только магнитное влияние, поскольку, как показано в [1], электростатическое влияние более чем в 50 раз менее интенсивно, чем магнитное при выполнении требований ПОТЭУ по установке заземления на отключенной ВЛ. Магнитное влияние в большей степени определяется продольной составляющей вектора напряженности электрического поля от влияющих линий $E_{x}$, которая без учета токов смещения определяется дифференциальным уравнением

$$
\frac{d^{2} E_{x}}{d y^{2}}+\frac{d^{2} E_{x}}{d z^{2}}-k_{3}^{2} E_{x}=0
$$

где $k_{3}=\sqrt{j \omega \mu_{0} \sigma_{3}}-$ волновое число земли; $\sigma_{3}$ - удельная проводимость грунта земли; $\omega-$ циклическая частота; $\mu_{0}$ - магнитная постоянная.

Решение уравнения при принятии допущения о горизонтальном расположении проводников влияющей и отключенной ВЛ можно представить в виде

$$
E_{x}=-j \frac{\omega \mu_{0}}{4 \pi} I\left[\ln \frac{(h+z)^{2}+y^{2}}{(h-z)^{2}+y^{2}}+4 \int_{0}^{\infty} \frac{e^{-v(h+z)}}{v+\sqrt{v^{2}+k_{3}^{2}}}\right],
$$

где $I$ - значения тока, протекающего по проводнику влияющей ЛЭП; $h$ - высота подвеса влияющего проводника ЛЭП над землей; $z, y$ - координаты отключенного провода ВЛ.

Входящий в (2) интеграл выражают через интеграл Карсона [3].

$$
\mathrm{I}_{k}=j\left[\int_{0}^{\infty} \frac{e^{-v(h+z)}}{v+\sqrt{v^{2}+k_{3}^{2}}} \cos y v d v .\right] .
$$

Зная $E_{x}$, однозначно определяем эквивалентную ЭДС, наводимую в проводнике отключенной ВЛ при прохождении ее параллельно влияющей ВЛ:

$$
E_{i}=E_{x} l,
$$


где $l$ - длина участка влияющего проводника, так же и в случае сходящихся влияющей ВЛ и отключенной ВЛ:

$$
E_{i}=\operatorname{ctg} \alpha \int_{y_{a}}^{y_{b}} E_{x} d y,
$$

где $\alpha$ - угол между влияющей и отключенной ВЛ; $y_{a}, y_{b}$ - минимальное и максимальное расстояния между влияющей и отключенной ВЛ на сходящемся участке.

В [3] показано, что с погрешностью, не превышающей 5 \%, интеграл Карсона (3) может быть представлен в виде

$$
\mathrm{I}_{k}=\frac{j}{4} \ln \frac{\left(h+z+\frac{2}{k_{3}}\right)^{2}+y^{2}}{(h+z)^{2}+y^{2}} .
$$

После подстановки $(2,6)$ в (4) получим соотношение для определения ЭДС, создаваемое магнитным полем (МП):

$$
E_{i}=-j \frac{\omega \mu_{0}}{4 \pi}\left[\ln \frac{(h+z)^{2}+y^{2}}{(h-z)^{2}+y^{2}}+\ln \frac{\left(h+z+\frac{2}{k_{3}}\right)^{2}+y^{2}}{(h+z)^{2}+y^{2}}\right] I_{i} l_{i}=-M_{i} I_{i} l_{i} .
$$

Более точное определение ЭДС, создаваемой магнитным влиянием, возможно произвести, зная распределение векторного магнитного потенциала $A$ от влияющей линии. Зная векторный магнитный потенциал, можно определить магнитный поток, пронизывающий некоторую площадь. Далее можно рассчитать собственную и взаимную индуктивность.

Для более точного расчета магнитного поля необходимо учитывать, что провод, жестко закрепленный в двух точках на разной высоте и испытывающий равномерно распределенную нагрузку как собственного веса, веса гололеда, так и давления ветра имеет стрелу провеса. Жесткость поперечного сечения проводов, используемых для транспорта электроэнергии, меньше равномерно распределенной нагрузки. Механическое напряжение в произвольной точке обусловлено только растяжением и направлено по касательной к кривой в рассматриваемой точке.

При расчете параметров магнитного поля, создаваемого влияющей линией электропередачи, в качестве математической модели реального проводника будем использовать уравнение идеально гибкой нити

$$
y=y_{\max } \operatorname{ch}\left(\frac{x}{y_{\max }}\right),
$$

где $y_{\max }$ - координата точки максимального провеса, м.

Уравнение (8) можно разложить в функциональный ряд Маклорена, который для пролетов длиной менее 700 м примет вид

$$
y=k x^{2}
$$

где $k$ - постоянная, зависящая от приведенной нагрузки на проводник и механических характеристик проводника.

$$
-349-
$$


Определим параметры МП с учетом провисания проводников линии электропередачи. Для этого получим выражение вектора магнитной индукции магнитного поля, создаваемого единственным проводом одного пролета. Величина тока, протекающего по проводнику, известна. Вектор магнитной индукции связан с векторным магнитным потенциалом

$$
\bar{B}=\operatorname{rot} \bar{A}
$$

Определим выражение для векторного магнитного потенциала. Для определения этого потенциала разобьем проводник на малые токовые участки $I d l$, касательные к проводу в точке $(x, y, z)$ (рис. 1). Этот токовый отрезок создает элементарный векторный потенциал $d \bar{A}:$

$$
d \bar{A}_{+}=\frac{\mu \mu_{0}}{4 \pi} \frac{I d \bar{l}}{r}
$$

где $\mu_{0}=4 \pi \cdot 10^{-7} \Gamma$ н/м - магнитная постоянная; $\mu$ - относительная магнитная проницаемость воздуха, в дальнейших расчетах примем ее равной единице.

Поскольку проводник находится над поверхностью идеальной земли, то ее влияние заменим зеркальным изображением $I^{\prime} d \bar{l}^{\prime}$, который создает в расчетной точке векторный потенциал $d \bar{A}^{\prime}$. Ток $I^{\prime}$ меньше по модулю тока $I$ на величину

$$
k=\frac{\mu_{\text {зем }}-\mu_{\text {воз }}}{\mu_{\text {зем }}+\mu_{\text {бозо }}}
$$

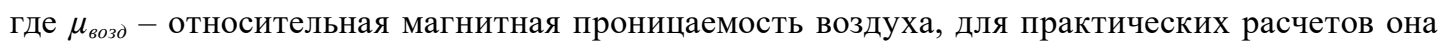
мало отличается от единицы; $\mu_{\text {зем }}$ - относительная магнитная проницаемость грунта земли.

При распространении электромагнитных волн промышленной частоты токи проводимости преобладают над токами смещения, поэтому происходит лишь незначительное ее проникновение в глубь земли. Следовательно, грунт для электромагнитных волн низкой частоты можно рассматривать как проводник. Таким образом, $k \rightarrow 1$, значит, $I^{\prime}=I$ и $d \bar{A}_{-}=\frac{\mu \mu_{0}}{4 \pi} \frac{I d \bar{l}^{\prime}}{r^{\prime}}$.

Результирующий векторный потенциал в точке $M$ от элементарного участка с током и его изображения определим по принципу суперпозиции $d \bar{A}=d \bar{A}_{+}+d \bar{A}_{-}=\bar{i} d A_{X}+\bar{j} d A_{Y}+\bar{k} d A_{Z}$.

Проекции векторного магнитного потенциала

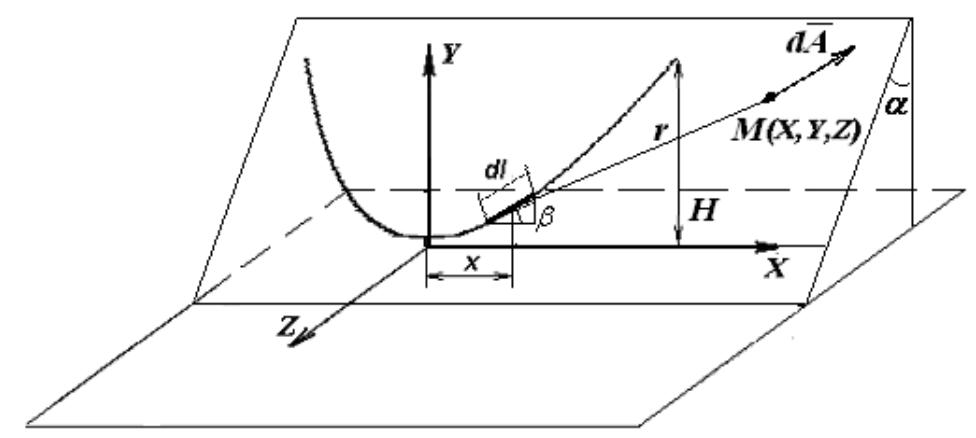

Рис. 1. К определению магнитной индукции от одного пролета

Fig. 1. To the magnetic field determination from the one span 


$$
\begin{gathered}
d A_{X}=\frac{\mu \mu_{0}}{4 \pi} I d l \cos \beta\left(\frac{1}{r}+\frac{1}{r^{\prime}}\right) ; d A_{Y}=\frac{\mu \mu_{0}}{4 \pi} I d l \sin \beta \cos \alpha\left(\frac{1}{r}-\frac{1}{r^{\prime}}\right) ; \\
d A_{Z}=\frac{\mu \mu_{0}}{4 \pi} I d l \sin \beta \sin \alpha\left(\frac{1}{r}+\frac{1}{r^{\prime}}\right),
\end{gathered}
$$

где $r$ - расстояние от токового элемента до точки $M(X, Y, Z)$

$$
r=\sqrt{(X-x)^{2}+(Y-y)^{2}+(Z-z)^{2}}
$$

$r^{\prime}$ - расстояние от изображения токового элемента до точки $M(X, Y, Z)$

$$
r^{\prime}=\sqrt{(X-x)^{2}+(Y+y)^{2}+(Z-z)^{2}} .
$$

Проекции векторного магнитного потенциала определим интегрированием по всей длине проводника:

на $O X=\frac{\mu \mu_{0}}{4 \pi} I \int_{-\frac{L}{2}}^{\frac{L}{2}}\left(\frac{1}{\left.\sqrt{\left((X-x)^{2}+\left(Y-\left(k x^{2}+h_{2}\right) \cos \alpha\right)^{2}+\left(Z-\left(H_{n o d}-k x^{2}-h_{2}\right) \sin \alpha\right)^{2}\right.}\right)}+\right.$

$$
\begin{aligned}
& \left.+\frac{1}{\left.\sqrt{(X-x)^{2}+\left(Y+\left(k x^{2}+h_{2}\right) \cos \alpha\right)^{2}+\left(Z-\left(H_{n o d}-k x^{2}-h_{2}\right) \sin \alpha\right)^{2}}\right)}\right) d x \\
& \text { на 0Y } A_{Y}=\frac{\mu \mu_{0}}{2 \pi} I k \cos \alpha \int_{-\frac{L}{2}}^{\frac{L}{2}}\left(\frac{x}{\left.\sqrt{\left((X-x)^{2}+\left(Y-\left(k x^{2}+h_{2}\right) \cos \alpha\right)^{2}+\left(Z-\left(H_{n o d}-k x^{2}-h_{2}\right) \sin \alpha\right)^{2}\right.}\right)}\right.
\end{aligned}
$$

$A_{Z}=\frac{\mu \mu_{0}}{2 \pi} I k \sin \alpha \int_{-\frac{L}{2}}^{\frac{L}{2}}\left(\frac{x}{\left.\sqrt{(X-x)^{2}+\left(Y-\left(k x^{2}+h_{2}\right) \cos \alpha\right)^{2}+\left(Z-\left(H_{n о д}-k x^{2}-h_{2}\right) \sin \alpha\right)^{2}}\right)^{2}}+\right.$

$$
-\frac{x}{\sqrt{\left((X-x)^{2}+\left(Y+\left(k x^{2}+h_{2}\right) \cos \alpha\right)^{2}+\left(Z-\left(H_{n o d}-k x^{2}-h_{2}\right) \sin \alpha\right)^{2}\right)}} d x
$$

$$
+\frac{x}{\left.\sqrt{\left((X-x)^{2}+\left(Y+\left(k x^{2}+h_{2}\right) \cos \alpha\right)^{2}+\left(Z-\left(H_{n o d}-k x^{2}-h_{2}\right) \sin \alpha\right)^{2}\right.}\right)} d x=
$$

В декартовой системе координат ротор имеет вид $\operatorname{rot} \overline{\mathrm{A}}=\left|\begin{array}{ccc}\bar{i} & \bar{j} & \bar{k} \\ \frac{\partial}{\partial x} & \frac{\partial}{\partial y} & \frac{\partial}{\partial z} \\ A_{x} & A_{y} & A_{z}\end{array}\right|$.

Следовательно, проекции вектора магнитной индукции

$$
B_{X}=\frac{\partial A_{z}}{\partial Y}-\frac{\partial A_{y}}{\partial Z} ; B_{Y}=\frac{\partial A_{x}}{\partial Z}-\frac{\partial A_{z}}{\partial X} ; B_{Z}=\frac{\partial A_{y}}{\partial X}-\frac{\partial A_{x}}{\partial Y} .
$$

Модуль магнитной индукции в произвольной точке

$$
B(M)=\sqrt{B_{X}^{2}+B_{Y}^{2}+B_{Z}^{2}} .
$$


На рис. 2 изображены зависимости вектора индукции магнитного поля $B(X$, const, const $)$ от расстояния вдоль единичного пролета $X$, на постоянной высоте $Y=1,8$ м, для различных расстояний до плоскости провеса провода $Z$, при допущении, что стрела провеса проводника вертикальна (ее координата $z=0$ ).

Рисунок 3 демонстрирует зависимости вектора индукции магнитного поля $B$ (const, const, Z) от расстояния до плоскости провода $Z$ при высоте расчетной точки 1,8 м

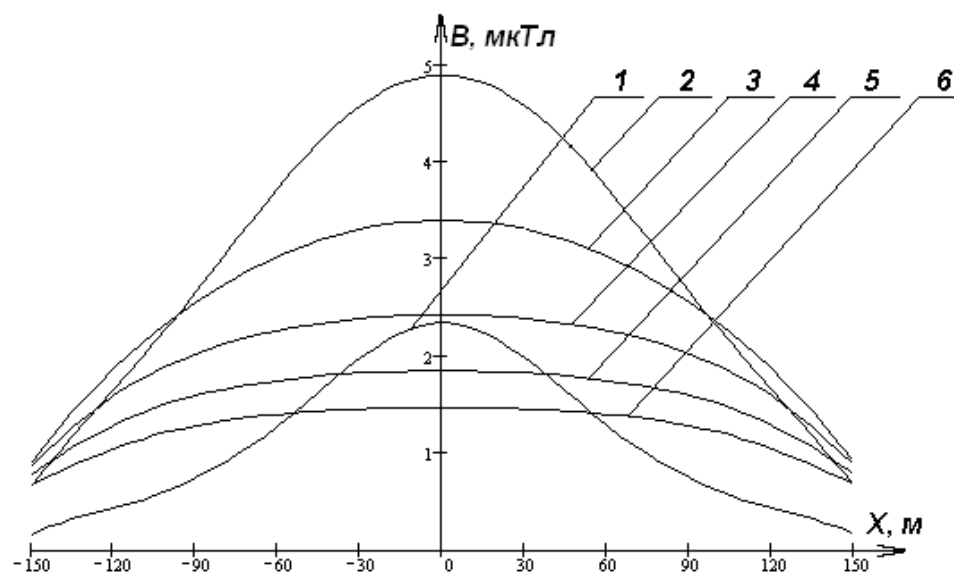

1) $Z=0 \mathrm{м}$; 2) $Z=10 \mathrm{м}$; 3) $Z=20 \mathrm{м}$; 4) $Z=30 \mathrm{м}$; 5) $Z=40 \mathrm{м}$; 6) $Z=50 \mathrm{м}$

Рис. 2. Зависимость вектора магнитной индукции вдоль единичного пролета от расстояния до плоскости провода на высоте $1,8 \mathrm{M}$

Fig. 2. The dependence of the magnetic induction vector along the single span on the distance to the wire plane at a height of $1.8 \mathrm{~m}$

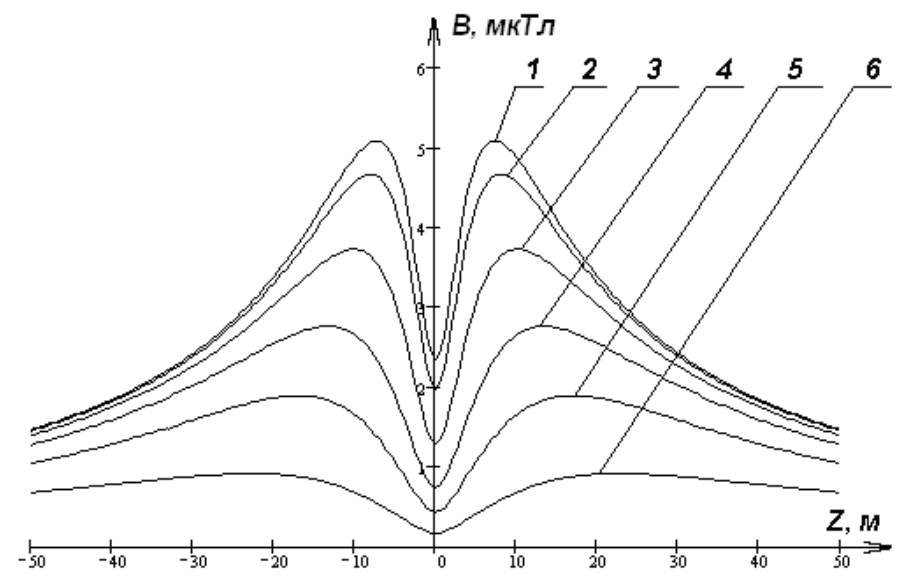

1) $X=0$ м; 2) $X=30$ м; 3) $X=60$ м; 4) $X=90$ м; 5) $X=120$; 6) $X=150$ м

Рис. 3. Зависимость вектора магнитной индукции от расстояния до плоскости провода, от расстояния вдоль единичного пролета на высоте 1,8 м

Fig. 3. The dependence of the of magnetic induction vector on the distance to the plane of the wire and on the distance along a single span at a height of $1.8 \mathrm{~m}$ 
для различных расстояний вдоль единичного пролета $X$ при допущении, что стрела провеса вертикальна.

Число пролетов необходимо учитывать при расчете вектора магнитной индукции магнитного поля.

На рис. 4 показано несколько кривых распределения индукции магнитного поля в случае учета магнитного поля нескольких смежных пролетов.

Из данных графика следует, что учет более трех пролетов (двух смежных) практически не влияет на точность расчетов. В качестве эталонных значений величины индукции магнитного поля $B_{э т}$ приняты такие, которые были получены при учете десяти смежных пролетов. Относительную погрешность $\varepsilon$ при этом рассчитаем как $\varepsilon=\frac{B_{э m}-B}{B_{э m}} 100 \%$, где $B$ - вектор магнитной индукции, вычисленный для числа пролетов меньше 21.

На рис. 5 изображена относительная погрешность индукции магнитного поля от расстояния до оси симметрии.

Зависимость $B(X)$ является периодической четной функцией с периодом, равным длине габаритного пролета [4]. После разложения в ряд Фурье и применяя численные методы вычисления интегралов, возможно определить коэффициенты $a$ :

$$
B(X)=\frac{a_{0}}{2}+\sum_{n=1}^{\infty} a_{n} \cos \frac{n \pi X}{l} .
$$

Поскольку параметры МП изменяются в зависимости от координаты $X$ и известно среднее значение на всем протяжении под пролетом, равное постоянному члену $B_{c p}=a_{0} / 2$, определим среднюю высоту подвеса проводника $H_{c p}$ для горизонтально расположенного проводника, который создает в расчетной точке такое же МП.

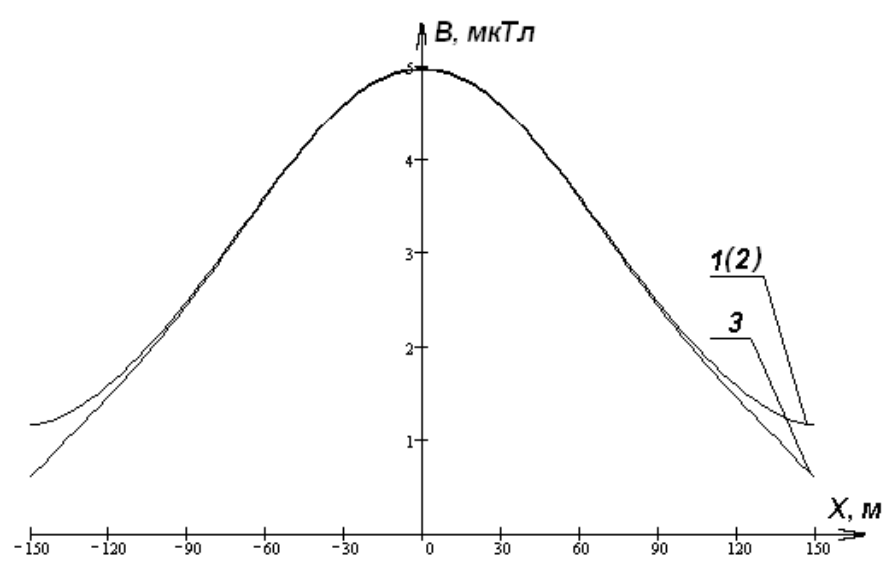

Рис. 4. Продольная эпюра распределения вектора магнитной индукции МП ВЛ при учете: 1) пяти пролетов; 2) трех пролетов; 3) одного пролета

Fig. 4. The longitudinal diagram of the overhead power line magnetic induction vector distribution 1) five spans; 2) three spans; 3) one span 


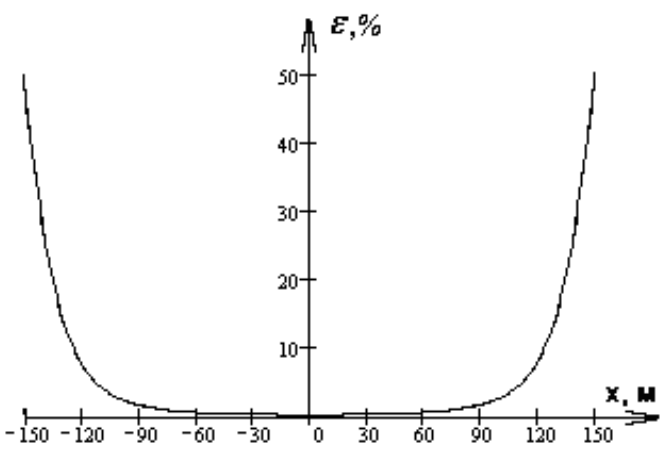

a)

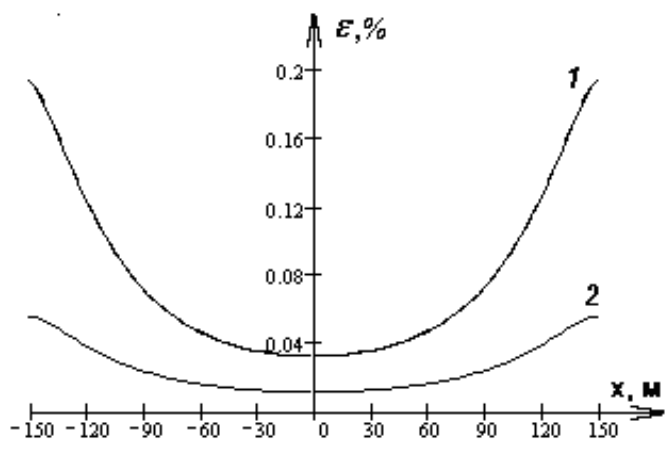

б)

Рис. 5. Относительная погрешность вектора магнитной индукции при учете: а) одного пролета; б) 1 трех пролетов, 2 - пяти пролетов

Fig. 5. The magnetic induction vector relative error considering: a) one span; б) 1 - three spans; 2 - five span

Индукция магнитного поля от горизонтального проводника с учетом влияния земли на поверхности земли и расстоянии $\mathrm{Z}$ от его оси $B_{c p}=2 \frac{\mu \mu_{0} I}{2 \pi} \frac{Z}{Z^{2}+H_{c p}^{2}}$.

Среднюю высоту подвеса провода определим, приравняв последнее выражение к постоянной составляющей $\mathrm{a}_{0} / 2$. Получим $H_{c p}=\sqrt{\frac{\mu \mu_{0} I Z}{\pi B_{c p}}-Z^{2}}$.

\section{Заключение}

Из анализа графиков, приведенных на рисунках, можно сделать следующие выводы:

- индукция магнитного поля максимальна не в плоскости нахождения провода, а на незначительном расстоянии от нее;

- на расстоянии, приблизительно равном трети длины габаритного пролета от провода, индукция МП уменышается не более чем в два раза от максимального значения;

- минимальных значений магнитная индукция достигает вблизи поверхности земли;

- при учете одного пролета минимальная погрешность составила 0,29 \% непосредственно под стрелой провеса; максимальная погрешность достигает 50 \% и наблюдается вблизи точек подвеса провода; с точностью $\approx 5$ \% влияние только одного пролета можно учитывать на 75 \% длины пролета;

- при учете влияния более двух смежных пролетов погрешность становится пренебрежимо малой, $\varepsilon \approx 0,2 \%$, и, следовательно, при расчетах, связанных с определением наведенного напряжения, достаточно учитывать только три пролета. 


\section{Список литературы}

[1] Цицикян Г.Н. Электромагнитная совместимость в электроэнергетике. СПб.: Элмор, 2007. 184 c. [Tsitsikyan G.N. The Electromagnetic compatibility in the electric power industry. SPb., Elmor, 2007. 184 p. (In Russian)]

[2] Белицын И.В., Котырло Т.В., Макаров А.В. Эллиптическое электрическое и магнитное поля электроустановок. Метод их расчета и нормирования. Известия Томского политехнического университета, 2008, 4, 61-65. [Belitsyn I.V., Kotyrlo T.V., Makarov A.V. The Elliptical electric and magnetic fields of electrical installations. Method of their calculation and rationing. Bulletin of the Tomsk Polytechnic University, 2008, 4, 61-65. (In Russian)]

[3] Перельман Л.С. Таблицы интегралов Карсона для использования в расчетах волновых процессов в линиях с учетом земли. Известия НИИ постоянного тока, 1965, 11, 342-360. [Perel'man L.S. The Carson integrals tables for use in calculations of the wave processes in power lines considering the Earth. Bulletin Direct Current Power Transmission Research Institute, 1965, 11, 342-360. (In Russian)]

[4] Belitsyn I.V., Khomutov S.O. Method of quality improving of electric energy by changing the topology of wires connection on overhead power transmission lines. International Journal of Applied Engineering Research, 2017, 12(3), 376-381/ 